\title{
THE NORM OF AN INFINITE L-MATRIX
}

\section{Ludovick Bouthat AND JAVAD MASHREGHi*}

Abstract. Evaluating the norm of infinite matrices, as operators acting on the sequence space $\ell^{2}$, is not an easy task. For a few celebrated matrices, e.g., the Hilbert matrix and the Cesàro matrix, the precise value of the norm is known. But, for many other important cases we use estimated values of norm. In this note, we study the norm of $L$-matrices $A=\left[a_{n}\right]$, which appear in studying Hadamard multipliers of function spaces. We provide some necessary and sufficient conditions for the finiteness of norm and study the sharpness of these conditions. In particular, for the decay rate $a_{n}=O\left(1 / n^{\alpha}\right)$, our characterization is complete. Finally, parallel to the above classical results of Hilbert and Cesàro, we succeed to show that $\left\|A_{s}\right\|=4$ for the family of $L$-matrices $A_{s}=[1 /(n+s)]$, irrelevant of the parameter $s$ which runs over $[1 / 2, \infty)$.

Mathematics subject classification (2010): 15A60, 15A04, 39B42.

Keywords and phrases: Operator norm, sequence spaces, infinite matrices.

\section{REFERENCES}

[1] Durmuş Bozkurt, On the $l_{p}$ norms of Hadamard product of Cauchy-Toeplitz and Cauchy-Hankel matrices, Linear and Multilinear Algebra, 45 (4): 333-339, 1999.

[2] Arlen Brown, P. R. Halmos And A. L. Shields, Cesàro operators, Acta Sci. Math. (Szeged), 26: 125-137, 1965.

[3] Man Duen ChoI, Tricks or treats with the Hilbert matrix, Amer. Math. Monthly, 90 (5): 301-312, 1983.

[4] Dan Dai, Mourad E. H. Ismail and Xiang-Sheng Wang, Doubly infinite Jacobi matrices revisited: resolvent and spectral measure, Adv. Math., 343:157-192, 2019.

[5] Paul Richard Halmos, A Hilbert space problem book, volume 19 of Graduate Texts in Mathematics, Springer-Verlag, New York-Berlin, second edition, 1982. Encyclopedia of Mathematics and its Applications, 17.

[6] G. H. Hardy, Divergent series, Éditions Jacques Gabay, Sceaux, 1992. With a preface by J. E. Littlewood and a note by L. S. Bosanquet, Reprint of the revised (1963) edition.

[7] David Hilbert, Ein Beitrag zur Theorie des Legendre'schen Polynoms, Acta Math., 18 (1): 155$159,1894$.

[8] Neil Hindman, Recent results on partition regularity of infinite matrices, In Connections in discrete mathematics, pages 200-213, Cambridge Univ. Press, Cambridge, 2018.

[9] Mourad E. H. Ismail AND FrantišEK ŠTAMPaCH, Spectral analysis of two doubly infinite Jacobi matrices with exponential entries, J. Funct. Anal., 276 (6): 1681-1716, 2019.

[10] JAVAd MAShreghi, Representation theorems in Hardy spaces, volume 74 of London Mathematical Society Student Texts, Cambridge University Press, Cambridge, 2009.

[11] JAVAD MASHREGHI AND THOMAS RANSFORD, Linear polynomial approximation schemes in Banach holomorphic function spaces, Anal. Math. Phys., 9 (2): 899-905, 2019.

[12] JOHN LINDSAY ORR, An estimate on the norm of the product of infinite block operator matrices, J. Combin. Theory Ser. A, 63 (2): 195-209, 1993.

[13] J. SCHUR, Bemerkungen zur Theorie der beschränkten Bilinearformen mit unendlich vielen Veränderlichen, J. Reine Angew. Math., 140: 1-28, 1911.

[14] SÜleyman SolaK, Research problem: on the norms of infinite Cauchy-Toeplitz-plus-CauchyHankel matrices, Linear Multilinear Algebra, 54 (6): 397-398, 2006. 
[15] Süleyman Solak and Durmuş Bozkurt, On the spectral norms of Cauchy-Toeplitz and Cauchy-Hankel matrices, Appl. Math. Comput., 140 (2-3): 231-238, 2003.

[16] C. V. M. VAN DER MEE AND S. SEATZU, A method for generating infinite positive self-adjoint test matrices and Riesz bases, SIAM J. Matrix Anal. Appl., 26 (4): 1132-1149, 2005.

[17] David M. Young,, Iterative solution of large linear systems, Academic Press, New York-London, 1971. 